Note

\section{High-boiling Constituents of Clove Oil (Eugenia caryophyllata ThunB.) and Hop Oil (Humulus lupulus LiNN.)}

\section{Toshikazu Uchida, Yoshiharu Matsubara and Akemi ADACHI}

Department of Applied Chemistry, Faculty of Science and Engineering, Kinki University, Kowakae, Higashiosaka-shi 577

Received January 16, 1986

Clove oil (Eugenia caryophyllata THUNB.) and hop oil (Humulus lupulus LiNN.) are used as materials for eugenol and beer. Of the oxygenated derivatives of $\beta$-caryophyllene found in their essential oils, there have been reported caryophyllene oxide, caryophylla-3(12), 7(13)-dien-6 $\alpha$-ol and caryophylla-3(12), 6-dien-4-ol in clove oil, ${ }^{1)}$ and caryophyllene oxide, ${ }^{1,2)}$ caryophyllene alcohol ${ }^{2)}$ and caryophylla-3(12), 6-dien-4-ol ${ }^{1)}$ in hop oil. Both clove and hop neutral oil contain $\beta$-caryophyllene as the major component in their high boiling fraction. Thus, it can be expected that the oxygenated derivatives of $\beta$-caryophyl- lene by a biosynthetic route may exist in their neutral essential oils. We have extensively examined these essential oils, using clove and hop oils supplied by the Takasago Perfumery Co., Ltd.

The removal of acids and phenols from the respective essential oils gave the neutral oils. More than 49 and 82 peaks were respectively observed in the gas chromatograms of these neutral oils (Figs. 1 and 2). As shown in Table I, four compounds were identified as sesquiterpene alcohols by comparing their GC-MS with those of authentic samples. The authentic samples were synthesized by well-established methods. Thus, oxidation of $\beta$-caryophyllene in acetic acid with lead tetraacetate followed by hydrolysis gave $45,{ }^{3)} 46\left(=71^{\prime}\right),{ }^{4)} 48\left(=73^{\prime}\right)^{4)}$ and $49\left(=75^{\prime}\right)^{4)}$ as minor products. These were purified by means of preparative GLC before use.

Compounds $46\left(=71^{\prime}\right), 48\left(=73^{\prime}\right)$ and $49\left(=75^{\prime}\right)$ have not previously been found in the essential oils of clove and hop, and 4,4-dimethyltricyclo[6.3.2.0 2,5]trideca-8-ene-1-ol (45) was shown to be present in the natural products for the first time.

\section{Apparatus}

Gas chromatography. We used a Yanaco model G 180TFP gas chromatograph equipped with a flame-ionization detector. A glass capillary column (inside diameter, $0.28 \mathrm{~mm}$; length, $20 \mathrm{~m}$ ) coated with PEG $20 \mathrm{M}$ was used. The column temperature was programed at $2^{\circ} \mathrm{C}$ per minute from 60 to $180^{\circ} \mathrm{C}$, and was held isothermally at $180^{\circ} \mathrm{C}$ for $0.5 \mathrm{hr}$. The inlet pressure for the nitrogen carrier gas was $1.0 \mathrm{~kg} / \mathrm{cm}^{2}$. The gas chromatogram was obtained

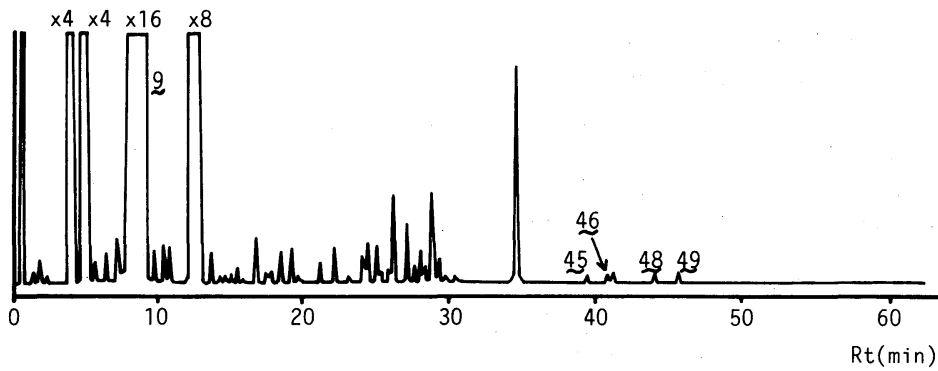

FIG. 1. Gas Chromatogram of Clove Oil.

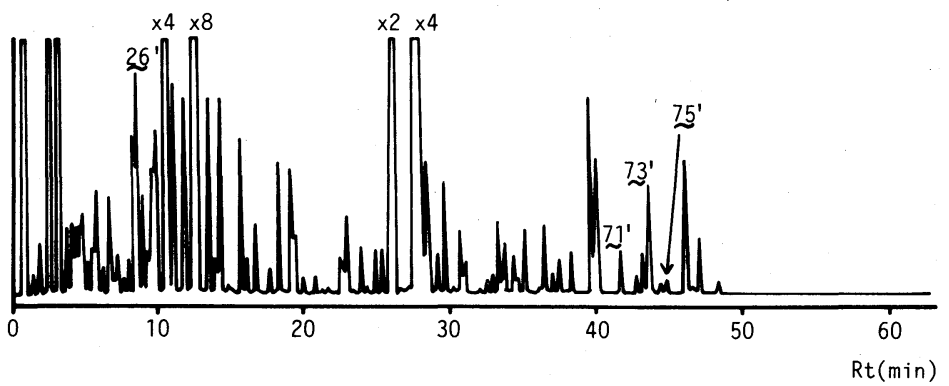

FIG. 2. Gas Chromatogram of Hop Oil. 


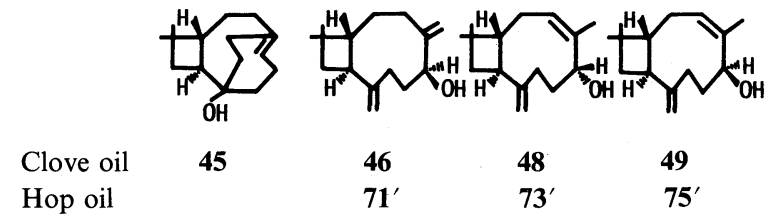

FIG. 3.

Table I. Sesquiterpene Components of Clove and Hop Oils

Clove oil

\begin{tabular}{|c|c|}
\hline Peak No. & Compound \\
\hline $45^{3)}$ & 4,4-Dimethyltricyclo[6.3.2. $\left.0^{2,5}\right]$ trideca-8-ene-1-ol \\
\hline \multirow[t]{2}{*}{$46^{4)}$} & Caryophylla-4(12),8(13)-dien-5 $\beta$-ol \\
\hline & (10,10-Dimethyl-2,6-dimethylenbicyclo[7.2.0]undecan-5 $\beta$-ol) \\
\hline \multirow[t]{2}{*}{$48^{4)}$} & Caryophylla-3,8(13)-dien-5 $\alpha$-ol \\
\hline & (11,11-Dimethyl-8-methylenbicyclo[7.2.0]undeca-3-ene-5 $\alpha$-ol) \\
\hline \multirow[t]{2}{*}{$49^{4)}$} & Caryophylla-3,8(13)-dien-5 $\beta$-ol \\
\hline & (11,11-Dimethyl-8-methylenbicyclo[7.2.0]undeca-3-ene-5 $\beta$-ol) \\
\hline
\end{tabular}

Hop oil

Peak No.

Compound

\begin{tabular}{|c|c|}
\hline $71^{\prime 4)}$ & $\begin{array}{l}\text { Caryophylla-4(12),8(13)-dien-5 } \beta \text {-ol } \\
(10,10 \text {-Dimethyl-2,6-dimethylenbicyclo[7.2.0]undecan-5 } \beta \text {-ol) }\end{array}$ \\
\hline $7^{\prime 4)}$ & $\begin{array}{l}\text { Caryophylla-3,8(13)-dien-5 } \alpha \text {-ol } \\
\text { (11,11-Dimethyl-8-methylenbicyclo[7.2.0]undeca-3-ene-5 } \alpha \text {-ol) }\end{array}$ \\
\hline $75^{\prime 4)}$ & $\begin{array}{l}\text { Caryophylla-3,8(13)-dien-5 } \beta \text {-ol } \\
\quad(11,11 \text {-Dimethyl-8-methylenbicyclo[7.2.0]undeca-3-ene-5 } \beta \text {-ol })\end{array}$ \\
\hline
\end{tabular}

under these conditions by injecting $0.4 \mu \mathrm{l}$ of neutral oil.

Mass spectrometry. Mass spectra were obtained on a JEOL JMS HX-100 mass spectrometer. The operating conditions were: ionization voltage, $70 \mathrm{eV}$; ion-accelerating voltage, $5000 \mathrm{~V}$; temperatures of the ionization chamber and sample injection block, 270 and $230^{\circ} \mathrm{C}$, respectively.

\section{MS spectral data}

45: $m / z 220\left(\mathbf{M}^{+}, 4 \%\right), 205\left(\mathbf{M}-\mathrm{CH}_{3}, 5\right), 202\left(\mathrm{M}-\mathrm{H}_{2} \mathrm{O}\right.$, 3), 191 (6), $187\left(\mathrm{M}-\mathrm{CH}_{3}-\mathrm{H}_{2} \mathrm{O}, 13\right), 177$ (8), 173 (4), 163 (7), $162(33), 159(11), 149(10), 147$ (48), $145(11), 136(11)$, 135 (48), 133 (19), 131 (18), 121 (22), 119 (38), 117 (8), 107 (52), 105 (33), 95 (12), 93 (40), 91 (49), 85 (10), 81 (15), 79 (33), 77 (24), 71 (28), 67 (26), 65 (12), 55 (26), 53 (19), 51 (6), 43 (100), 41 (48), 39 (20).

46 and 71': $m / z 220\left(\mathrm{M}^{+}, 1 \%\right), 205\left(\mathrm{M}-\mathrm{CH}_{3}, 3\right), 202$ $\left(\mathrm{M}-\mathrm{H}_{2} \mathrm{O}, 2\right), 187\left(\mathrm{M}-\mathrm{CH}_{3}-\mathrm{H}_{2} \mathrm{O}, 6\right), 177$ (6), 159 (11), 149 (9), 136 (100), 123 (12), 121 (13), 119 (15), $117(13), 109$ (22), 107 (20), 105 (21), 95 (19), 93 (25), 91 (30), 81 (25), 79 (28), 77 (16), 69 (38), 67 (20), 55 (22), 53 (13), 43 (9), 41 (48), 39 (14).

48 and $7^{\prime}: m / z$ 220( $\left.\mathbf{M}^{+}, 9 \%\right), 205\left(\mathbf{M}-\mathrm{CH}_{3}, 13\right), 202$ $\left(\mathrm{M}-\mathrm{H}_{2} \mathrm{O}, 11\right), 187\left(\mathrm{M}-\mathrm{CH}_{3}-\mathrm{H}_{2} \mathrm{O}, 16\right), 177(10), 173$ (9), $164(14), 160(15), 149(20), 146(26), 135(20), 133(34)$,
131 (26), 123 (79), $121(33), 119(18), 117(11), 109(37), 107$ (58), 105 (42), 97 (11), 95 (48), 93 (94), 91 (50), 84 (56), 81 (100), 79 (55), 77 (33), 71 (39), 69 (43), 67 (37), 65 (14), 55 (36), 53 (23), 43 (12), 41 (88), 39 (25).

49 and $75^{\prime}: m / z 220\left(\mathrm{M}^{+}, 6 \%\right), 205\left(\mathrm{M}-\mathrm{CH}_{3}, 14\right), 202$ $\left(\mathrm{M}-\mathrm{H}_{2} \mathrm{O}, 14\right), 187\left(\mathrm{M}-\mathrm{CH}_{3}-\mathrm{H}_{2} \mathrm{O}, 46\right), 177$ (9), 173 (10), 164 (17), 161 (40), 159 (34), 151 (29), 149 (33), 147 (40), 135 (42), 133 (47), 131 (49), 123 (72), 119 (33), 117 (18), 109 (79), 107 (82), 105 (67), 95 (13), 93 (93), 91 (74), 81 (74), $79(64), 77(48), 69(43), 67(43), 59(10), 55(61), 53$ (37), 43 (30), 41 (100), 39 (36).

\section{REFERENCES}

1) H. Iwamuro, H. Takenokuchi, Y. Matsubara and Y. Iizuka, Agric. Biol. Chem., 47, 2099 (1983).

2) Y. Naya and M. Kotake, Nippon Kagaku Zasshi, 91, 275 (1970).

3) Y. Matsubara, T. Uchida and T. Ono, The 29th Symposium of Chemistry of Terpenes, Essential Oils and Aromatics of Japan, 1985, p. 42.

4) M. Kasano and Y. Matsubara, Nippon Kagaku Kaishi, 1978, 1170. 